\title{
A Lempel-Ziv Text Index on Secondary Storage
}

\author{
Diego Arroyuelo and Gonzalo Navarro \\ Dept. of Computer Science, University of Chile, \\ Blanco Encalada 2120, Santiago, Chile. \\ \{darroyue, gnavarro\}@dcc.uchile.cl
}

\begin{abstract}
Full-text searching consists in locating the occurrences of a given pattern $P[1 . . m]$ in a text $T[1 . . u]$, both sequences over an alphabet of size $\sigma$. In this paper we define a new index for full-text searching on secondary storage, based on the Lempel-Ziv compression algorithm and requiring $8 u H_{k}+o(u \log \sigma)$ bits of space, where $H_{k}$ denotes the $k$-th order empirical entropy of $T$, for any $k=o\left(\log _{\sigma} u\right)$. Our experimental results show that our index is significantly smaller than any other practical secondary-memory data structure: 1.4-2.3 times the text size including the text, which means $39 \%-65 \%$ the size of traditional indexes like String $B$-trees [Ferragina and Grossi, JACM 1999]. In exchange, our index requires more disk access to locate the pattern occurrences. Our index is able to report up to 600 occurrences per disk access, for a disk page of 32 kilobytes. If we only need to count pattern occurrences, the space can be reduced to about 1.04-1.68 times the text size, requiring about 20-60 disk accesses, depending on the pattern length.
\end{abstract}

\section{Introduction and Previous Work}

Many applications require to store huge amounts of text, which need to be searched to find patterns of interest. Full-text searching is the problem of locating the occ occurrences of a pattern $P[1 . . m]$ in a text $T[1 . . u]$. Both the text and the pattern are modeled as sequences of symbols over a finite alphabet $\Sigma$ of size $\sigma$. Unlike word-based text searching, we wish to find any text substring, not only whole words or phrases. This has applications in texts where the concept of word does not exist or is not well defined, such as in DNA or protein sequences, Oriental languages texts such as Chinese or Japanese, MIDI pitch sequences, program code, etc. There exist two classical kind of queries, namely: $(1) \operatorname{count}(T, P)$ : counts the number of occurrences of pattern $P$ in $T$; $(2)$ locate $(T, P)$ : reports the starting positions of the occ occurrences of pattern $P$ in $T$.

Usually in practice the text is a very long sequence (of several of gigabytes, or even terabytes) which is known beforehand, and we want to locate (or count) the pattern occurrences as fast as possible. Thus, we preprocess $T$ to build a data structure (or index), which is used to speed up the search, avoiding a sequential scan. However, by using an index we increase the space requirement. This is

\footnotetext{
* Supported in part by CONICYT PhD Fellowship Program (first author) and Fondecyt Grant 1-050493 (second author).
} 
unfortunate when the text is very large. Traditional indexes like suffix trees [1] require $O(u \log u)$ bits to operate; in practice this space can be 10 times the text size with a very careful implementation [2], and so the index does not fit entirely in main memory even for moderate-size texts. In these cases the index must be stored on secondary memory and the search proceeds by loading the relevant parts into main memory.

Text compression is a technique to represent a text using less space. We denote by $H_{k}$ the $k$-th order empirical entropy of a sequence of symbols $T$ over an alphabet of size $\sigma$ [3]. The value $u H_{k}$ provides a lower bound to the number of bits needed to compress $T$ using any compressor that encodes each symbol considering only the context of $k$ symbols that precede it in $T$. It holds that $0 \leqslant H_{k} \leqslant H_{k-1} \leqslant \cdots \leqslant H_{0} \leqslant \log \sigma$ (log means $\log _{2}$ in this paper).

To provide fast access to the text using little space, the current trend is to use compressed full-text self-indexes, which allows one to search and retrieve any part of the text without storing the text itself, while requiring space proportional to the compressed text size (e.g., $O\left(u H_{k}\right)$ bits) $[4,5]$. Therefore we replace the text with a more space-efficient representation of it, which at the same time provides indexed access to the text. This has applications in cases where we want to reduce the space requirement by not storing the text, or when accessing the text is so expensive that the index must search without having the text at hand, as occurs with most Web search engines. As compressed self-indexes replace the text, we are also interested in operations display $(T, P, \ell)$, which displays a context of $\ell$ symbols surrounding the pattern occurrences and extract $(T, i, j)$, which decompresses the substring $T[i . . j]$, for any text positions $i \leqslant j$.

The use of a compressed full-text self-index may totally remove the need to use the disk. However, some texts are so large that their corresponding indexes do not fit entirely in main memory, even compressed. Unlike what happens with sequential text searching, which speeds up with compression because the compressed text is transferred faster to main memory [6], working on secondary storage with a compressed index usually requires more disk accesses in order to find the pattern occurrences. Yet, these indexes require less space, which in addition can reduce the seek time incurred by a larger index because seek time is roughly proportional to the size of the data.

We assume a model of computation where a disk page of size $B$ (able to store $b=\omega(1)$ integers of $\log u$ bits, i.e. $B=b \log u$ bits) can be transferred to main memory in a single disk access. Because of their high cost, the performance of our algorithms is measured as the number of disk accesses performed to solve a query. We count every disk access, which is an upper bound to the real number of accesses to solve the problem, as we disregard the disk caching due to the operating system. In this model we can hold a constant number of disk pages in main memory. We assume that our text $T$ is static, i.e., there are no insertions nor deletions of text symbols.

There are not many works on full-text indexes on secondary storage, which definitely is an important issue. One of the best known indexes for secondary memory is the String B-tree [7], although this is not a compressed data structure. 
It requires (optimal) $O\left(\log _{b} u+\frac{m+o c c}{b}\right)$ disk accesses in searches and (worst-case optimal) $O(u / b)$ disk blocks of space. This value is, in practice, about 12.5 times the text size (not including the text) [8], which is prohibitive for very large texts.

Clark and Munro [9] present a representation of suffix trees on secondary storage (the Compact Pat Trees, or CPT for short). This is not a compressed index, and also needs the text to operate. Although not providing worst-case guarantees, the representation is organized in such a way that the number of disk accesses is reduced to $3-4$ per query. The authors claim that the space requirement of their index is comparable to that of suffix arrays, needing about 4-5 times the text size (not including the text).

Mäkinen et al. [10] propose a technique to store a Compressed Suffix Array on secondary storage, based on backward searching [11]. This is the only proposal to store a (zero-th order) compressed full-text self-index on secondary memory, requiring $u\left(H_{0}+O(\log \log \sigma)\right)$ bits of storage and a counting cost of at most $2\left(1+m\left\lceil\log _{B} u\right\rceil\right)$ disk accesses. Locating the occurrences of the pattern would need $O(\log u)$ extra accesses per occurrence.

In this paper we propose a version of Navarro's LZ-index [12] that can be efficiently handled on secondary storage. Our index requires $8 u H_{k}+o(u \log \sigma)$ bits of space for $k=o\left(\log _{\sigma} u\right)$. In practice the space requirement is about 1.42.3 times the text size including the text, which is significantly smaller than any other practical secondary-memory data structure. Although we cannot provide worst-case guarantees at search time (just as in [9]), our experiments show that our index is effective in practice, although requiring more disk accesses than larger indexes: our LZ-index is able to report up to 600 pattern occurrences per disk access. On the other hand, count queries can be performed requiring about 20-60 disk accesses (depending on the pattern length).

\section{The LZ-index Data Structure}

Assume that the text $T[1 . . u]$ has been compressed using the LZ78 [13] algorithm into $n+1$ phrases $T=B_{0} \ldots B_{n}$ (see Appendix A for details on Lempel-Ziv compression). We say that $i$ is the phrase identifier of phrase $B_{i}$. The data structures that conform the LZ-index are [12]:

1. LZTrie: is the trie formed by all the LZ78 phrases $B_{0} \ldots B_{n}$. Given the properties of LZ78 compression, this trie has exactly $n+1$ nodes, each one corresponding to a string.

2. RevTrie: is the trie formed by all the reverse strings $B_{0}^{r} \ldots B_{n}^{r}$. In this trie there could be empty nodes not representing any block.

3. Node: is a mapping from block identifiers to their node in LZTrie.

4. RNode: is a mapping from block identifiers to their node in RevTrie.

Each of these four structures requires $n \log n(1+o(1))$ bits if they are represented succinctly. As $n \log u=u H_{k}+O(k n \log \sigma) \leqslant u \log \sigma$ for any $k$ [14], the final size of the LZ-index is $4 u H_{k}+o(u \log \sigma)$ bits of space for any $k=o\left(\log _{\sigma} u\right)$. 
To search for a pattern $P[1 . . m]$, we distinguish three types of occurrences of $P$ in $T$, depending on the phrase layout [12]. For locate queries, each pattern occurrence is reported in the format $\llbracket t$, off set $\rrbracket$, where $t$ is the phrase where the occurrence starts, and off set is the distance between the beginning of the occurrence and the end of the phrase. However, the pattern occurrences can be shown as text positions with little extra effort [15].

Occurrences of Type 1. The occurrence lies inside a single phrase (there are $o c c_{1}$ occurrences of this type). Given the properties of LZ78, every phrase $B_{k}$ containing $P$ is formed by a shorter phrase $B_{\ell}$ concatenated to a symbol $c$. If $P$ does not occur at the end of $B_{k}$, then $B_{\ell}$ contains $P$ as well. We want to find the shortest possible phrase $B_{i}$ in the LZ78 referencing chain for $B_{k}$ that contains the occurrence of $P$. Since phrase $B_{i}$ has the string $P$ as a suffix, $P^{r}$ is a prefix of $B_{i}^{r}$, and can be easily found by searching for $P^{r}$ in RevTrie. Say we arrive at node $v$. Any node $v^{\prime}$ descending from $v$ in RevTrie (including $v$ itself) corresponds to a phrase terminated with $P$. Thus we traverse and report all the subtrees of the LZTrie nodes corresponding to each $v^{\prime}$. Total locate time is $O\left(m+o c c_{1}\right)$.

Occurrences of Type 2. The occurrence spans two consecutive phrases, $B_{k}$ and $B_{k+1}$, such that a prefix $P[1 . . i]$ matches a suffix of $B_{k}$ and the suffix $P[i+$ 1..m] matches a prefix of $B_{k+1}$ (there are $o c c_{2}$ occurrences of this type). $P$ can be split at any position, so we have to try them all. For every possible split $P[1 . . i]$ and $P[i+1 . . m]$ of $P$, assume the search for $P^{r}[1 . . i]$ in RevTrie yields node $v_{r e v}$, and the search for $P[i+1 . . m]$ in LZTrie yields node $v_{l z}$. Then, we check each phrase $t$ in the subtree of $v_{\text {rev }}$ and report occurrence $\llbracket t, i \rrbracket$ if $N o d e[t+1]$ descends from $v_{l z}$. Each such check takes constant time. Yet, if the subtree of $v_{l z}$ has fewer elements, we do the opposite: check phrases from $v_{l z}$ in $v_{r e v}$, using $R$ Node $[t-1]$. The total time is proportional to the smallest subtree size among $v_{r e v}$ and $v_{l z}$.

Occurrences of Type 3. The occurrence spans three or more phrases, $B_{k-1} \ldots$ $B_{\ell+1}$, such that $P[i . . j]=B_{k} \ldots B_{\ell}, P[1 . . i-1]$ matches a suffix of $B_{k-1}$ and $P[j+$ 1..m] matches a prefix of $B_{\ell+1}$ (there are $o c c_{3}$ occurrences of this type). Since the LZ78 algorithm guarantees that every phrase represents a different string there is at most one phrase matching $P[i . . j]$ for each choice of $i$ and $j$. Thus, occ 3 is limited to $O\left(\mathrm{~m}^{2}\right)$ occurrences. We first identify the only possible phrase matching every substring $P[i . . j]$. This is done by searching for every pattern substring in LZTrie, recording in a matrix $C_{l z}[i, j]$ the LZTrie node corresponding to $P[i . . j]$. Then we try to find the $O\left(\mathrm{~m}^{2}\right)$ maximal concatenations of successive phrases that match contiguous pattern substrings. If $P[i . . j]=B_{k} . . B_{\ell}$ is a maximal concatenation, then we check whether phrase $B_{\ell+1}$ starts with $P[j+1 . . m]$, that is, we check whether Node $[\ell+1]$ is a descendant of node $C_{l z}[j+1, m]$. Finally we check whether phrase $B_{k-1}$ ends with $P[1 . . i-1]$, by starting from Node $[i-1]$ in LZTrie and successively going to the parent to check whether the last $i-1$ 
nodes, read backwards, equal $P^{r}[1 . . i-1]$. If all these conditions hold, then we report an occurrence $\llbracket k-1, i-1 \rrbracket$. Overall locate time is $O\left(m^{2} \log m\right)$ worst-case and $O\left(m^{2}\right)$ on average.

\section{LZ-index on Secondary Storage}

The LZ-index [12] was originally designed to work in main memory, and hence it has a non-regular pattern of access to the index components. As a result, it is not suitable to work on secondary storage. In this section we show how to achieve locality in the access to the components of the LZ-index, so as to have good secondary storage performance. In this process we introduce some redundancy over main-memory proposals $[12,15]$.

\subsection{Solving the Basic Trie Operations}

To represent the tries of the index we use a space-efficient representation similar to the hierarchical representation of [16], which now we make searchable. We cut the trie into disjoint blocks of size $B$ such that every block stores a subtree of the whole trie. We arrange these blocks in a tree by adding some inter-block pointers, and thus the trie is represented by a tree of subtrees.

We cut the trie in a bottom-up fashion, trying to maximize the number of nodes in each block. This is the same partition used by Clark and Munro [9], and so we also suffer of very small blocks. To achieve a better fill ratio and reduce the space requirement, we store several trie blocks into each disk page.

Every trie node $x$ in this representation is either a leaf of the whole trie, or it is an internal node. For internal nodes there are two cases: the node $x$ is internal to a block $p$ or $x$ is a leaf of block $p$ (but not a leaf of the whole trie). In the latter case, $x$ stores a pointer to the representation $q$ of its subtree. The leaf is also stored as a fictitious root of $q$, so that every block is a subtree. Therefore, every such node $x$ has two representations: (1) as a leaf in block $p$; (2) as the root node of the child block $q$.

Each block $p$ of $N$ nodes and root node $x$ consists basically of:

- the balanced parentheses (BP) representation [17] of the subtree, requiring $2 N+o(N)$ bits;

- a bit-vector $F_{p}[1 . . N]$ (the flags) such that $F_{p}[j]=1$ iff the $j$-th node of the block (in preorder) is a leaf of $p$, but not a leaf of the whole trie. In other words, the $j$-th node has a pointer to the representation of its subtree. We represent $F_{p}$ using the data structure of [18] to allow rank and select queries in constant time and requiring $N+o(N)$ bits;

- the sequence $\operatorname{let}_{p}[1 . . N]$ of symbols labeling the arcs of the subtree, in preorder. The space requirement is $N\lceil\log \sigma\rceil$ bits;

- only in the case of LZTrie, the sequence $i d s_{p}[1 . . N]$ of phrase identifiers in preorder. The space requirement is $N \log n$ bits.

- a pointer to the leaf representation of $x$ in the parent block; 
- the depth and preorder of $x$ within the whole trie;

- a variable number of pointers to child blocks. The number of child blocks of a given block can be known from the number of $1 \mathrm{~s}$ in $F_{p}$.

- an array Size $_{p}$ such that each pointer to child block stores the size of the corresponding subtree.

Using the information stored in each trie block, we are able to compute the basic operations: parent $(x)$ (which gets the parent of node $x$ ), $\operatorname{child}(x, \alpha)$ (which yields the child of node $x$ by label $\alpha$ ), depth $(x)$ (which gets the depth of node $x$ in the trie), subtreesize (x) (which gets the size of the subtree of node $x$, including $x$ itself), preorder $(x)$ (which gets the preorder number of node $x$ in the trie), and ancestor $(x, y)$ (which tells us whether node $x$ is ancestor of node $y$ ). Operations subtreesize, depth, preorder, and ancestor can be computed without extra disk accesses, while operations parent and child require one disk access in the worst case. In Appendix B we explain how to compute them.

Analysis of Space Complexity. In the case of LZTrie, as the number of nodes is $n$, the space requirement is $2 n+n+n \log \sigma+n \log n+o(n)$ bits, for the $\mathrm{BP}$ representation, the flags, the symbols, and phrase identifiers respectively. To this we must add the space required for the inter-block pointers and the extra data added to each block, such as the depth of the root, etc. If the trie is represented by a total of $K$ blocks, these data add up to $O(K \log n)$ bits. The bottom-up partition of the trie ensures $K=O(n / b)$, so the extra data requires $O\left(\frac{n}{b} \log n\right)$ bits. As $b=\omega(1)$, this space is $o(n \log n)=o(u \log \sigma)$ bits.

In the case of RevTrie, as there can be empty nodes, we represent the trie using a Patricia tree [19], compressing empty unary paths so that there are $n \leqslant$ $n^{\prime} \leqslant 2 n$ nodes. In the worst case the space requirement is $4 n+2 n+2 n \log \sigma+o(n)$ bits, plus the extra information as before.

As we pack several trie blocks in a disk page, we ensure a utilization ratio of $50 \%$ at least. Hence the space of the tries can be at most doubled on disk.

\subsection{Navigating the Tries}

As we explained in Section 2, we need to search for every pattern substring $P[i . . j](1 \leqslant i \leqslant j \leqslant m)$ in LZTrie and for $P^{r}[1 . . i](1 \leqslant i \leqslant m)$ in RevTrie. We first consider the search in LZTrie. For every $i=1 \ldots m$, we search for $P[i . . j]$ for increasing $j$. Besides matrix $C_{l z}$ defined in Section 2, we fill an array $N[1 . . m]$ storing information about the search on LZTrie. As we can hold the root block of LZTrie always in main memory, we start searching for $P[1 . . j]$ until we arrive at a leaf of the block. At this point we store in $N[1]$ a pointer to the block of LZTrie where to continue the search, along with the value of $j$ that produced the disk access. Then we search for $P[2 . . j]$, and in the same way store the information in $N[2]$, and so on until we search for every substring of $P$ in the root block.

At this point we start again from $i=1$ and access the block pointed by $N[1]$, until we eventually need a new disk access. We then traverse $N$ looking for an entry of the array pointing to the same disk page so as to continue that search 
too using the disk page we have just read (recall that we store several trie blocks in each disk page). In this way we try to use each disk access to a maximum.

For each substring found we store in $C_{l z}$ the phrase identifier, preorder, and subtree size of the corresponding node. This is useful to avoid further accesses later.

We use part of the work already done in LZTrie to reduce the work in RevTrie. Let $j$ be the maximum value such that $P[1 . . j]$ is a string in LZTrie (i.e., it is a LZ78 phrase). Hence, $P^{r}[1 . . j]$ is a string in RevTrie. Also, for every $j^{\prime \prime}=1 \ldots j, P^{r}\left[1 . . j^{\prime \prime}\right]$ is a string in RevTrie (because of Property 1 of Appendix A). Then we search for every $P^{r}\left[1 . . j^{\prime \prime}\right]$ in RevTrie in a Patricia tree fashion, but as such string is present in the trie we are sure that, after consuming all of $P^{r}\left[1 . . j^{\prime \prime}\right]$ in the descent, we have arrived to the right node, without the need of the final check needed by Patricia trees [19] (which would need to access LZTrie to retrieve the string, as we do not store the text). To descend we use the same procedure as in LZTrie. The other phrases $P\left[1 . . j^{\prime}\right]$ for $j^{\prime} \geqslant j$ cannot be found using the LZTrie, since they could be represented by an empty node in RevTrie or by a position in a string between two nodes. We look for $P\left[1 . . j^{\prime}\right]$ as in a Patricia tree, this time requiring the final check, accessing the LZTrie to retrieve the string. We use an array $C_{r}[1 . . m]$ such that $C_{r}[j]$ stores information about the RevTrie preorder and subtree size of the RevTrie node corresponding to $P^{r}[1 . . j]$.

\subsection{Reducing the Navigation between Structures}

We add the following data structures with the aim of reducing the number of disk accesses required by the LZ-index at search time, in order to perform locate, count, extract, and display queries:

- $\operatorname{Pre}_{l z}[1 . . n]$ : a mapping from phrase identifiers to the corresponding LZTrie preorder, requiring $n \log n$ bits of space.

- Rev[1..n]: a mapping from RevTrie preorder positions to the corresponding LZTrie node, requiring $n \log u+n$ bits of space. Later in this section we explain why we need this space.

- TPos $\operatorname{Pos}_{l z}[1 . . n]$ : if the phrase corresponding to the node with preorder $i$ in LZTrie starts at position $j$ in the text, then $T \operatorname{Pos}_{l z}[i]$ stores the value $j$. This array requires $n \log u$ bits and is used to locate the text positions of pattern occurrences.

- $L R[1 . . n]$ : an array requiring $n \log n$ bits. If the node with preorder $i$ in LZTrie corresponds to the LZ78 phrase $B_{k}$, then $L R[i]$ stores the preorder of the RevTrie node for $B_{k-1}$.

- $S_{r}[1 . . n]$ : an array requiring $n \log u$ bits, storing in $S_{r}[i]$ the subtree size of the LZTrie node corresponding to the $i$-th RevTrie node (in preorder). This array is used for counting.

- Node[1..n]: the mapping from phrase identifiers to the corresponding LZTrie node, requiring $n \log n$ bits. This is used to solve extract and display queries. 
As the size of these arrays depends on the compressed text size, we do not need that much space to store them: they require $3 n \log u+3 n \log n+n$ bits, which summed to the tries gives $8 u H_{k}+o(u \log \sigma)$ bits, for any $k=o\left(\log _{\sigma} u\right)$.

If the index is used only for count queries, we basically need arrays $\operatorname{Pre}_{l z}$, $L R, S_{r}$, and the tries, plus an array $R L[1 . . n]$, which is similar to $L R$ but mapping from a RevTrie node for $B_{k}$ to the LZTrie preorder for $B_{k+1}$. All these add up to $6 u H_{k}+o(u \log \sigma)$ bits.

We explain now how to find the pattern occurrences. Assume that we have already searched for all the pattern substrings $P[i . . j]$ in LZTrie and all reversed prefixes $P^{r}[1 . . i]$ in RevTrie (see Section 3.2).

Occurrences of Type 1. Assume that the search for $P^{r}$ in RevTrie yields node $v_{r}$. For every node with preorder $i$, such that $\operatorname{preorder}\left(v_{r}\right) \leqslant i \leqslant \operatorname{preorder}\left(v_{r}\right)+$ subtreesize $\left(v_{r}\right)$ in RevTrie, with Rev[i] we get the node $v_{l z_{i}}$ in LZTrie representing a phrase $B_{t}$ ending with $P$. Note that the length of $B_{t}$ is $d=\operatorname{depth}\left(v_{l z_{i}}\right)$, and that the occurrence starts at position $d-m$ inside $B_{t}$. Therefore, if $p=$ preorder $\left(v_{l z_{i}}\right)$, the exact text position can be computed as $T \operatorname{Pos}_{l z}[p]+d-m$. We then traverse all the subtree of $v_{l z_{i}}$ and report, as an occurrence of type 1 , each node contained in this subtree, accessing TPos $l_{l z}\left[p . . p+\right.$ subtreesize $\left.\left(v_{l z_{i}}\right)\right]$ to find the text positions. Note that the offset $d-m$ stays constant for all nodes in the subtree.

Note that every node in the subtree of $v_{r}$ (or every $i$ in the above interval) produces a random access in LZTrie. In the worst case, the subtree of $v_{l z_{i}}$ has only one element to report ( $v_{l z_{i}}$ itself), and hence we have $o c c_{1}$ random accesses in the worst case. To reduce the worst case to $o c c_{1} / 2$, we use the $n$ extra bits in Rev: in front of the $\log u$ bits of each Rev element, a bit indicates whether we are pointing to a LZTrie leaf. In such a case we do not perform a random access to LZTrie, but we use the corresponding $\log u$ bits to store the exact text position of the occurrence.

To avoid accessing the same LZTrie page more than once, even for different trie blocks stored in that page, for each $\operatorname{Rev}[i]$ we solve all the other $\operatorname{Rev}[j]$ that need to access the same LZTrie page. As the tries are small, many random accesses could need to access the same page.

For count queries we traverse the $S_{r}$ array instead of Rev, summing up the sizes of the corresponding LZTrie subtrees without accessing them, therefore requiring $O\left(\right.$ occ $\left._{1} / b\right)$ disk accesses.

Occurrences of Type 2. For occurrences of type 2 we consider every possible partition $P[1 . . i]$ and $P[i+1 . . m]$ of $P$. Suppose the search for $P^{r}[1 . . i]$ in RevTrie yields node $v_{r}$ (with preorder $p_{r}$ and subtree size $s_{r}$ ), and the search for $P[i+$ 1..m] in LZTrie yields node $v_{l z}$ (with preorder $p_{l z}$ and subtree size $s_{l z}$ ). Then we traverse sequentially $L R[j]$, for $j=p_{l z} . . p_{l z}+s_{l z}$, reporting an occurrence at text position $T \operatorname{Pos}_{l z}[j]-i$ iff $L R[j] \in\left[p_{r} . . p_{r}+s_{r}\right]$. This algorithm has the nice property of traversing arrays $L R$ and $T P_{o s}$ sequentially, yet the number of elements traversed can be arbitrarily larger than $o c c_{2}$. 
For count queries, since we have also array $R L$, we choose to traverse $R L[j]$, for $j=p_{r} . . p_{r}+s_{r}$, when the subtree of $v_{r}$ is smaller than that of $v_{l z}$, counting an occurrence only if $R L[j] \in\left[p_{l z} . p_{l z}+s_{l z}\right]$.

To reduce the number of accesses from $2\left\lceil\frac{s_{l z}+1}{b}\right\rceil$ to $\left\lceil\frac{2\left(s_{l z}+1\right)}{b}\right\rceil$, we interleave arrays $L R$ and $T \operatorname{Pos}_{l z}$, such that we store $L R[1]$ followed by $T \operatorname{Pos}_{l z}[1]$, then $L R[2]$ followed by $T P \operatorname{Pos}_{l z}[2]$, etc.

Occurrences of Type 3. We find all the maximal concatenations of phrases using the information we got in Section 3.2. If we found that $P[i . . j]=B_{k}, \ldots, B_{\ell}$ is a maximal concatenation, we check whether phrase $B_{\ell+1}$ has $P[j+1 . . m]$ as a prefix, and whether phrase $B_{k-1}$ has $P[1 . . i-1]$ as a suffix. Note that, according to the LZ78 properties, $B_{\ell+1}$ starting with $P[j+1 . . m]$ implies that there exists a previous phrase $B_{t}, t<\ell+1$, such that $B_{t}=P[j+1 . . m]$. In other words, $C_{l z}[j+1, m]$ must not be null (i.e., phrase $B_{t}$ must exist) and the phrase identifier stored at $C_{l z}[j+1, m]$ must be less than $\ell+1$ (i.e., $t<\ell+1$ ). If these conditions hold, we check whether $P^{r}[1 . . i-1]$ exists in RevTrie, using the information stored at $C_{r}[i-1]$. Only if all these condition hold, we check whether $\operatorname{Pre}_{l z}[\ell+1]$ descends from the LZTrie node corresponding to $P[j+1 . . m]$ (using the preorder and subtree size stored at $\left.C_{l z}[j+1, m]\right)$, and if we pass this check, we finally check whether $L R\left[\operatorname{Pre}_{l z}[k]\right]$ (which yields the RevTrie preorder of the node corresponding to phrase $k-1$ ) descend from the RevTrie node for $P^{r}[1 . . i-1]$ (using the preorder and subtree size stored at $C_{r}[i-1]$ ). Fortunately, we have a high probability that $\operatorname{Pre}_{l z}[\ell+1]$ and $\operatorname{Pre}_{l z}[k]$ need to access the same disk page. If we find an occurrence, the corresponding position is $T \operatorname{Pos}_{l z}\left[\operatorname{Pre}_{l z}[k]\right]-(i-1)$.

Solving extract Queries. For extract $(T, i, j)$ we have the following problem: given any text position $i$, we need to compute the corresponding LZTrie node so as to extract the text from there. We store in a $B$-tree the starting text position for every phrase $B_{p \cdot h}$, for $p=1, \ldots, \frac{n}{h} h>0$. This requires $\frac{n}{h} \log u$ bits of space. We hold the root block of the $B$-tree always in main memory. Using the $B$-tree we can search for the rightmost phrase $B_{k}$ with starting text position $p_{k}$, such that $p_{k} \leqslant i$ and $p_{k}$ has been stored in the $B$-tree. From $p_{k}$ we get the disk page at which we access Node, using the fact that the sampling in the text positions is regular. We then repeatedly access Node $[k+s]$ in LZTrie, for $s \geqslant 0$, adding depth(Node $[k+s])$ to $p_{k}$, so as to get the text position $p_{k+s}$ of phrase $B_{k+s}$, until the sum is greater than $i$. At this point, we are in the LZTrie node corresponding to the phrase $B_{k+s}$ containing text position $i$. The text can be extracted by going successively to the parent in LZTrie, getting the symbol at each node. Once we reach the LZTrie root, we go on to extract the text in the following phrase. For any $h=\omega(1)$ we have $o(u \log \sigma)$ extra bits, and we perform $\omega(1)$ extra disk accesses.

For display $(T, P, \ell)$ queries, we first solve locate $(T, P)$. Then, for each occurrence starting at position $i$ we carry out extract $(T, i-\ell, i+m+\ell-1)$. 


\section{Experimental Results}

For the experiments of this paper we consider two text files: the text WsJ (Wall Street Journal) from the TREC collection [20], of 128 megabytes, and the XML file provided in the PizzaECChili Corpus ${ }^{1}$, downloadable from http://pizzachili. dcc.uchile.cl/texts/xml/dblp.xml.200MB.gz, of 200 megabytes. We searched for 5,000 random patterns, of length from 5 to 50 , generated from these files. As in [8], we assume a disk page size of 32 kilobytes. We compared our results against the following state-of-the-art indexes for secondary storage:

Suffix Arrays (SA): following [21] we divide the suffix array into blocks of $h \leqslant b$ elements (pointers to text suffixes), and move to main memory the first $l$ text symbols of the first suffix of each block, i.e. there are $\frac{u}{h} l$ extra symbols. We assume in our experiments that $l=m$ holds, which is the best situation. At search time, we carry out two binary searches [22] to delimit the interval $[i . . j]$ of the pattern occurrences. Yet, the first part of the binary search is done over the samples without accessing the disk. Once the blocks where $i$ and $j$ lie are identified, we bring them to main memory and finish the binary search, this time accessing the text on disk at each comparison. Therefore, the total cost is $2+2 \log h$ disk accesses. We must pay $\left\lceil\frac{o c c}{b}\right\rceil$ extra accesses to report the occurrences of $P$ within those two positions. The space requirement including the text is $\left(5+\frac{m}{h}\right)$ times the text size.

String B-trees [7]: in [8] they pointed out that an implementation of String $B$-trees for static texts would require about $2+\frac{2.125}{k}$ times the text size (where $k>0$ is a constant) and the height $h$ of the tree is 3 for texts of up to 2 gigabytes, since the branching factor (number of children of each tree node) is $b^{\prime} \approx \frac{b}{8.25}$. The experimental number of disk accesses given by the authors is $O(\log k)\left(\left\lfloor\frac{m}{b}\right\rfloor+2 h\right)+\left\lceil\frac{o c c}{b^{\prime}}\right\rceil$. We assume a constant of 1 for the $O(\log k)$ factor, since this is not clear in the paper [8, Sect. 2.1] (this is optimistic). We use $k=2,4,8,16$, and 32 .

Compact Pat Trees (CPT) [9]: we assume that the tree has height 3, according to the experimental results of Clark and Munro. We need $1+\left\lfloor\frac{o c c}{b}\right\rfloor$ extra accesses to locate the pattern occurrences. The space is about $4-5$ times the text size (plus the text).

We restrict our comparison to indexes that have been implemented, or at least simulated, in the literature. Hence we exclude the Compressed Suffix Arrays (CSAs) [10] since we only know that it needs at most $2\left(1+m\left\lceil\log _{b} u\right\rceil\right)$ accesses for count queries. This index requires about 0.22 and 0.45 times the text size for the XML and WSJ texts respectively, which, as we shall see, is smaller than ours. However, CSAs require $O(\log u)$ accesses to report each pattern occurrence ${ }^{2}$.

Fig. 1 shows the time/space trade-offs of the different indexes for count queries, for patterns of length 5 and 15. As it can be seen, our LZ-index requires

\footnotetext{
${ }^{1}$ http://pizzachili.dcc.uchile.cl

2 The work [23] extends this structure to achieve fast locate. The secondary-memory version is still a theoretical proposal and it is hard to predict how will it perform, so we cannot meaningfully compare it here.
} 

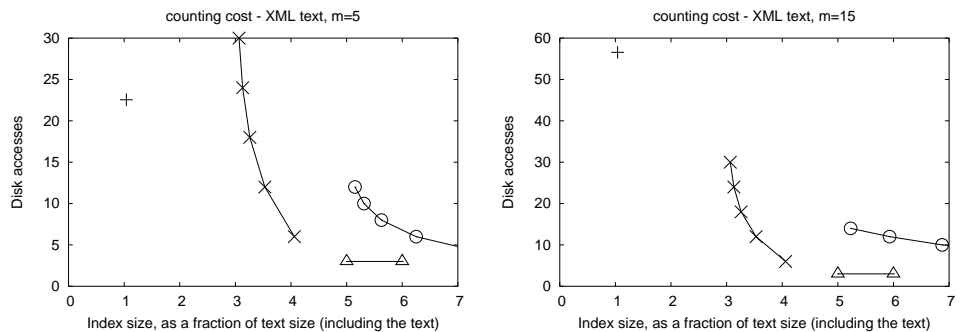

counting cost - WSJ text, $m=5$
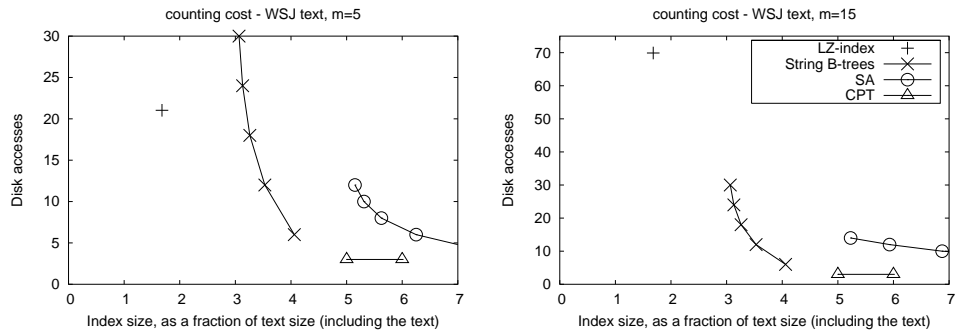

Fig. 1. Count cost vs. space requirement for the different indexes we tested.

about 1.04 times the text size for the (highly compressible) XML text, and 1.68 times the text size for the WSJ text. For $m=5$, the counting requires about 23 disk accesses, and for $m=15$ it needs about 69 accesses. Note that for $m=5$, there is a difference of 10 disk accesses among the LZ-index and String B-trees, the latter requiring 3.39 (XML) and 2.10 (WSJ) times the space of the LZindex. For $m=15$ the difference is greater in favor of String B-Trees. The SA outperforms the LZ-index in both cases, the latter requiring about $20 \%$ of SA. Finally, the LZ-index needs (depending on the pattern length) about 7-23 times the number of accesses of CPTs, the latter requiring 4.9-5.8 (XML) and 3-3.6 (WSJ) times the space of LZ-index.

Fig. 2 shows the time/space trade-offs for locate queries, this time showing the average number of occurrences reported per disk access. The LZ-index requires about 1.37 (XML) and 2.23 (WSJ) times the text size. The LZ-index is able of reporting about 597 (XML) and 63 (WSJ) occurrences per disk access for $m=5$, and about 234 (XML) and 10 (WSJ) occurrences per disk access for $m=15$. The average number of occurrences found for $m=5$ is 293,038 (XML) and 27,565 (WSJ); for $m=15$ there are 45,087 and 870 pattern occurrences on average. String B-trees report 3,449 (XML) and 1,450 (WSJ) occurrences per access for $m=5$, and for $m=15$ the results are 1,964 (XML) and 66 (WSJ) occurrences per access, while requiring 2.57 (XML) and 1.58 (WSJ) times the space of the LZ-index.

Fig. 3 shows the cost for the different parts of the LZ-index search algorithm, in the case of the XML text (WSJ yields similar results): the work done in the tries (labeled "tries"), the different types of occurrences, and the total cost ("total"). The total cost can be decomposed in three components: a part linear 

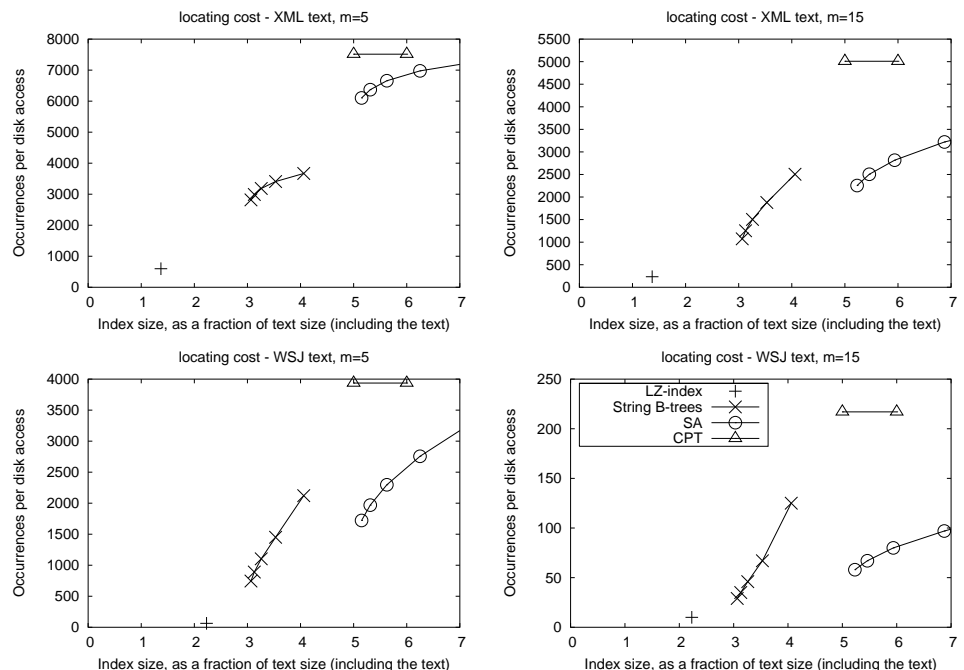

Fig. 2. Locate cost vs. space requirement for the different indexes we tested. Higher means better locate performance.
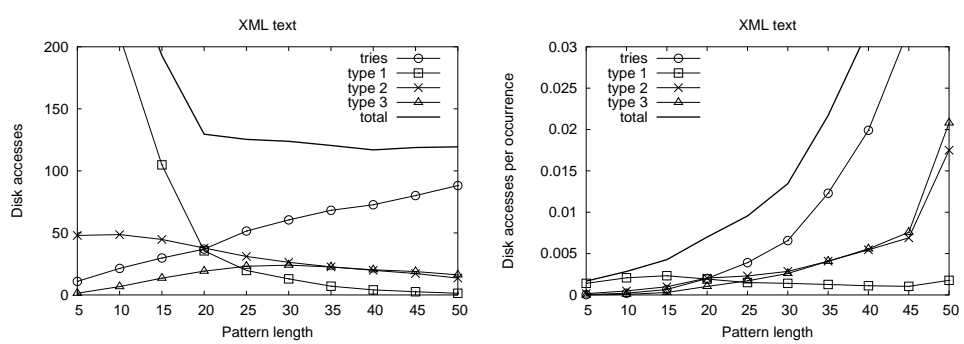

Fig. 3. Cost for the different parts of the LZ-index search algorithm.

on $m$ (trie traversal), a part linear in occ (type 1), and a constant part (type 2 and 3 ).

\section{Conclusions and Further Work}

The LZ-index [12] can be adapted to work on secondary storage, requiring up to $8 u H_{k}+o(u \log \sigma)$ bits of space, for any $k=o\left(\log _{\sigma} u\right)$. In practice, this value is about 1.4-2.3 times the text size, including the text, which means $39 \%-65 \%$ the space of String B-trees [7]. Saving space in secondary storage is important not only by itself (space is very important for storage media of limited size, such as CD-ROMs), but also to reduce the high seek time incurred by a larger index, which usually is the main component in the cost of accessing secondary storage, and is roughly proportional to the size of the data. 
Our index is significantly smaller than any other practical secondary-memory data structure. In exchange, it requires more disk accesses to locate the pattern occurrences. For XML text, we are able to report (depending on the pattern length) about 597 occurrences per disk access, versus 3,449 occurrences reported by String B-trees. For English text (WSJ file from [20]), the numbers are 63 vs. 1,450 occurrences per disk access. In many applications, it is important to find quickly a few pattern occurrences, so as to find the remaining while processing the first ones, or on user demand (think for example in Web search engines). Fig. 3 (left, see the line "tries") shows that for $m=5$ we need about 11 disk accesses to report the first pattern occurrence, while String B-trees need about 12. If we only want to count the pattern occurrences, the space can be dropped to $6 u H_{k}+o(u \log \sigma)$ bits; in practice 1.0-1.7 times the text size. This means $29 \%-48 \%$ the space of String B-trees, with a slowdown of 2-4 in the time.

We have considered only number of disk accesses in this paper, ignoring seek times. Random seeks cost roughly proportionally to the size of the data. If we multiply number of accesses by size of the indexes, we get a very rough idea of the overall seek times. We can see that the smaller size of our LZ-index should favor it in practice. For example, it is very close to String B-trees for counting on XML and $m=5$ (Fig. 1). This product model is optimistic, but counting only accesses is pessimistic.

We leave for future work the problems of handling dynamism (i.e., allowing insertion and deletions of texts) and the direct construction on secondary storage, adapting the method of [16] to work on disk.

\section{References}

1. Apostolico, A.: The myriad virtues of subword trees. In: Combinatorial Algorithms on Words. NATO ISI Series, Springer-Verlag (1985) 85-96

2. Kurtz, S.: Reducing the space requeriments of suffix trees. Softw. Pract. Exper. 29(13) (1999) 1149-1171

3. Manzini, G.: An analysis of the Burrows-Wheeler transform. JACM 48(3) (2001) 407-430

4. Navarro, G., Mäkinen, V.: Compressed full-text indexes. ACM Computing Surveys (2007) To appear.

5. Ferragina, P., Manzini, G.: Indexing compressed texts. JACM 54(4) (2005) 552581

6. Moura, E., Navarro, G., Ziviani, N., Baeza-Yates, R.: Fast and flexible word searching on compressed text. ACM TOIS 18(2) (2000) 113-139

7. Ferragina, P., Grossi, R.: The String B-tree: a new data structure for string search in external memory and its applications. JACM 46(2) (1999) 236-280

8. Ferragina, P., Grossi, R.: Fast string searching in secondary storage: theoretical developments and experimental results. In: Proc. SODA. (1996) 373-382

9. Clark, D., Munro, J.I.: Efficient suffix trees on secondary storage. In: Proc. SODA. (1996) 383-391

10. Mäkinen, V., Navarro, G., Sadakane, K.: Advantages of backward searching efficient secondary memory and distributed implementation of compressed suffix arrays. In: Proc. ISAAC. (2004) 681-692 
11. Sadakane, K.: Succinct representations of $l c p$ information and improvements in the compressed suffix arrays. In: Proc. SODA. (2002) 225-232

12. Navarro, G.: Indexing text using the Ziv-Lempel trie. J. of Discrete Algorithms 2(1) (2004) 87-114

13. Ziv, J., Lempel, A.: Compression of individual sequences via variable-rate coding. IEEE TIT 24(5) (1978) 530-536

14. Kosaraju, R., Manzini, G.: Compression of low entropy strings with Lempel-Ziv algorithms. SIAM J.Comp. 29(3) (1999) 893-911

15. Arroyuelo, D., Navarro, G., Sadakane, K.: Reducing the space requirement of LZ-index. In: Proc. CPM. (2006) 319-330

16. Arroyuelo, D., Navarro, G.: Space-efficient construction of LZ-index. In: Proc. ISAAC. (2005) 1143-1152

17. Munro, I., Raman, V.: Succinct representation of balanced parentheses and static trees. SIAM J.Comp. 31(3) (2001) 762-776

18. Munro, I.: Tables. In: Proc. FSTTCS. LNCS 1180 (1996) 37-42

19. Morrison, D.R.: Patricia - practical algorithm to retrieve information coded in alphanumeric. JACM 15(4) (1968) 514-534

20. Harman, D.: Overview of the third text REtrieval conference. In: Proc. Third Text REtrieval Conference (TREC-3), NIST Special Publication 500-207 (1995)

21. Baeza-Yates, R., Barbosa, E.F., Ziviani, N.: Hierarchies of indices for text searching. Inf. Systems 21(6) (1996) 497-514

22. Manber, U., Myers, G.: Suffix arrays: A new method for on-line string searches. SIAM J.Comp. 22(5) (1993) 935-948

23. González, R., Navarro, G.: Compressed text indexes with fast locate. In: Proc. of CPM'07. LNCS (2007) To appear.

24. Benoit, D., Demaine, E., Munro, I., Raman, R., Raman, V., Rao, S.: Representing trees of higher degree. Algorithmica 43(4) (2005) 275-292

25. Hon, W.K., Sadakane, K., Sung, W.K.: Succinct data structures for searchable partial sums. In: Proc. ISAAC. (2003) 505-516

\section{A Lempel-Ziv Compression}

The Ziv-Lempel compression algorithm of 1978 (usually named LZ78 [13]) is based on a dictionary of phrases, in which we add every new phrase computed. At the beginning of the compression, the dictionary contains a single phrase $b_{0}$ of length 0 . The current step of the compression is as follows: If we assume that a prefix $T[1 . . j]$ of $T$ has been already compressed into a sequence of phrases $Z=$ $b_{1} \ldots b_{r}$, all of them in the dictionary, then we look for the longest prefix of the rest of the text $T[j+1 \ldots u]$ which is a phrase of the dictionary. Once we have found this phrase, say $b_{s}$ of length $\ell_{s}$, we construct a new phrase $b_{r+1}=\left(s, T\left[j+\ell_{s}+1\right]\right)$, write the pair at the end of the compressed file $Z$, i.e. $Z=b_{1} \ldots b_{r} b_{r+1}$, and add the phrase to the dictionary.

We will call $B_{i}$ the string represented by phrase $b_{i}$, thus $B_{r+1}=B_{s} T[j+$ $\left.\ell_{s}+1\right]$. In the paper we assume that the text $T$ has been compressed using the LZ78 algorithm into $n+1$ phrases, $T=B_{0} \ldots B_{n}$, such that $B_{0}=\varepsilon$ (the empty string). We say that $i$ is the phrase identifier corresponding to $B_{i}, 0 \leqslant i \leqslant n$.

Property 1. For all $1 \leqslant k \leqslant n$, there exist $\ell<k$ and $c \in \Sigma$, such that $B_{k}=B_{\ell} \cdot c$. 
That is, every phrase $B_{k}$ (except $B_{0}$ ) is formed by a previous phrase $B_{\ell}$ plus a symbol $c$ at the end. This implies that the set of phrases is prefix closed, meaning that any prefix of a phrase $B_{k}$ is also an element of the dictionary. Therefore, a natural way to represent the set of strings $B_{0}, \ldots, B_{n}$ is a trie, which we call LZTrie.

Property 2. Every phrase $B_{i}, 0 \leqslant i<n$, represents a different text substring.

This property is used in the LZ-index search algorithm (see Section 2). The only exception to this property is the last phrase $B_{n}$. We deal with the exception by appending to $T$ a special symbol "\$" $\notin \Sigma$, assumed to be different to any other symbol in the alphabet. The last phrase will contain this symbol and thus will be unique too.

Definition 1. Let $b_{r}=\left(r_{1}, c_{1}\right), b_{r_{1}}=\left(r_{2}, c_{2}\right), b_{r_{2}}=\left(r_{3}, c_{3}\right)$, and so on until $r_{k}=0$ be phrases of the LZ78 parsing of $T$. The sequence of phrase identifiers $r, r_{1}, r_{2}, \ldots$ is called the referencing chain starting at phrase $r$.

The referencing chain starting at phrase $r$ reproduces the way phrase $b_{r}$ is formed from previous phrases, and is obtained by successively moving to the parent in the LZTrie.

The compression algorithm is $O(u)$ time in the worst case and efficient in practice provided we use the LZTrie, which allow rapid searching of the new text prefix (for each symbol of $T$ we move once in the trie). The decompression needs to build the same dictionary (the pair that defines the phrase $r$ is read at the $r$-th step of the algorithm).

Property 3 ([13]). It holds that $\sqrt{u} \leqslant n \leqslant \frac{u}{\log _{\sigma} u}$. Thus, $n \log u \leqslant u \log \sigma$ always holds.

Lemma 1 ([14]). It holds that $n \log u=u H_{k}+O(k n \log \sigma)$ for any $k$.

In our work we assume $k=o\left(\log _{\sigma} u\right)$, and hence $n \log u=u H_{k}+o(u \log \sigma)$.

\section{B Solving the Trie Operations}

Now we explain how to solve the basic operations needed at search time. In all cases suppose that node $x$ is the $j$-th node (in preorder) of block $p$.

1. Operation parent $(x)$, gets the parent of node $x$ and is undefined if $x$ is the root of the whole trie. Otherwise, if $x$ is not the root of some trie block, then both $x$ and parent $(x)$ are stored in the same block and parent $(x)$ is found in constant time using the $\mathrm{BP}$ representation. Otherwise, parent $(x)$ is stored in the parent block $q$ of $p$ (the block which is pointing to $p$ ). We follow the pointer to the leaf representation of $x$ in $q$ and finally use the parent operation on the $\mathrm{BP}$ representation of $q$. As a result, in the worst case we need one disk access to solve the operation. 
2. Operation $\operatorname{child}(x, \alpha)$, yields the child of node $x$ with label $\alpha$ and is undefined if $x$ is a leaf of the whole trie. Otherwise, if $x$ is not a leaf of some block, the desired child is also stored in block $p$. Therefore, we use the $\operatorname{child}(x, i)$ operation (which gets the $i$-th child of node $x$ ) provided by BP for $i=$ $1, \ldots, \sigma$, until we reach the child by symbol $\alpha$ (or until we discover that such child does not exist). This takes $O(\sigma) \mathrm{CPU}$ time in the worst case, but it is free of disk accesses. If $x$ is a leaf of block $p$, the child we are looking for is a child of the root representation of $x$ in the corresponding child block $q$. If $i=\operatorname{rank}_{1}\left(F_{p}, j\right)$, then $q$ is the $i$-th child block of $p$. We then move to $q$ and look for the child of its root by label $\alpha$. Hence, in the worst case we need only one disk access to solve this operation.

3. Operation $\operatorname{depth}(x)$ gets the depth of node $x$ in the trie. It can be computed as the depth of the root of $p$ plus the depth of $x$ within $p$, which can be computed in constant time by using BP [17]. Therefore, this operation can be computed in constant time and free of disk accesses.

4. Operation subtreesize $(x)$ gets the size of the subtree of node $x$, including $x$ itself. The BP representation allows us to solve the subtreesize operation in constant time, so the operation can be solved trivially if the subtree of $x$ is completely contained in block $p$ (we call subtreesize $e_{p}$ that operation). However, the subtree of $x$ can span more than its block. We use array Size to solve this problem. The portion of Size $e_{p}$ corresponding to $x$ goes from position $p_{1}=\operatorname{rank}_{1}\left(F_{p}, j-1\right)+1$ to $p_{2}=\operatorname{rank}_{1}\left(F_{p}, j+\operatorname{subtreesize}_{p}(x)-\right.$ $1)$. If $p_{1}$ equals $p_{2}$, the subtree of $x$ is completely stored in $p$. Otherwise, subtreesize $(x)$ can be computed as subtreesize $p_{p}(x)+\sum_{i=p_{1}}^{p_{2}}$ Size $_{p}[i]$. We do not need extra accesses to compute this operation.

5. Operation preorder $(x)$ gets the preorder number of node $x$ in the trie and is computed from three main components: the preorder of the root of block $p$ within the whole trie, plus the preorder of $x$ within $p$ (computed using BP), plus the number of nodes stored in child blocks of $p$, such that all nodes in these child blocks have preorder less than $x$. We compute the last term as $\sum_{i=1}^{r} \operatorname{Size}_{p}[i]$, where $r=\operatorname{rank}_{1}\left(F_{p}, j-1\right)$.

6. Operation ancestor $(x, y)$ tells us whether node $x$ is ancestor of node $y$ and is trivially solved provided we can solve operations subtreesize and preorder: node $x$ is an ancestor of node $y$ iff $\operatorname{preorder}(x) \leqslant \operatorname{preorder}(y) \leqslant \operatorname{preorder}(x)$ + subtreesize $(x)$.

Achieving Constant CPU Time. To achieve $O(1)$ CPU time we represent the subtrees in each block using the DFUDS representation [24] (which allows child $(x, \alpha)$ in $O(1)$ time), and represent Size $_{p}$ using a searchable partial sum data structure [25]. For a given $i$, these data structures allow one to compute, among others, operations $\operatorname{Size}_{p}[i]$, which retrieves the $i$-th value stored in the array, and $\operatorname{Sum}\left(\operatorname{Size}_{p}, i\right)$, which computes $\sum_{j=1}^{i} \operatorname{Size}_{p}[j]$, both in constant time and requiring $o\left(\mid\right.$ Size $\left._{p} \mid \log n\right)$ extra bits [25], if $\mid$ Size $_{p} \mid$ is the number of elements in array Size $_{p}$. 